\title{
One blind and three targeted searches for (sub)millisecond pulsars
}

\author{
E. Davoust ${ }^{1}$, G. Petit ${ }^{2}$, and T. Fayard ${ }^{3}$
}

1 IRAP, Université de Toulouse, CNRS, 14 avenue Edouard Belin, 31400 Toulouse, France e-mail: emmanuel. davoust@ast.obs-mip.fr

2 BIPM, Pavillion de Breteuil, 92312 Sèvres Cedex, France e-mail: gpetit@bipm.org

3 CNES/GRGS, 14 avenue Edouard Belin, 31400 Toulouse, France

e-mail: thierry. fayard@cnes.fr

Received 31 May 2011 / Accepted 21 July 2011

\begin{abstract}
Context. Millisecond pulsars are very useful for determining the properties of neutron stars, for testing General Relativity and for detecting gravitational waves. However, the number of known millisecond pulsars is very small compared to that of ordinary pulsars. Aims. We conducted one blind and three targeted searches for millisecond and submillisecond pulsars at radio frequencies.

Methods. The blind search was conducted within $3^{\circ}$ of the Galactic plane and at longitudes between $20^{\circ}$ and $110^{\circ}$. It takes 22073 pointings to cover this region, and 5487 different positions in the sky (i.e. $25 \%$ of the total) were actually observed. The first targeted search was aimed at Galactic globular clusters, the second one at 24 bright polarized and pointlike radiosources with steep spectra, and the third at 65 faint polarized and pointlike radiosources. The observations were conducted at the large radiotelescope of Nançay Observatory, at a frequency near $1400 \mathrm{MHz}$, the exact value depending on the backend. Two successive backends were used, first a VLBI S2 system, second a digital acquisition board and a PC with large storage capacity sampling the signal at $50 \mathrm{Mb} / \mathrm{s}$ on one bit, over a 24-MHz band and in one polarization. The bandwidth of acquisition of the second backend was later increased to $48 \mathrm{MHz}$ and the sampling rate to $100 \mathrm{Mb} / \mathrm{s}$. The survey used the three successive setups, with respective sensitivities of 3.5, 2.2, and $1.7 \mathrm{mJy}$. The targeted-search data were obtained with the third setup and reduced with a method based on the Hough transform, yielding a sensitivity of $0.9 \mathrm{mJy}$. The processing of the data was done in slightly differed time by soft-correlation in all cases.

Results. No new short-period millisecond pulsars were discovered in the different searches. To better understand the null result of the blind survey, we estimate the probability of detecting one or more short-period pulsars among a given Galactic population of synthetic pulsars with our setup: $25 \%$ for the actual incomplete survey and $79 \%$ if we had completed the whole survey with a uniform nominal sensitivity of $1.7 \mathrm{mJy}$. The alternative of surveying a smaller, presumably more densely populated, region with a higher sensitivity would have a low return and would be impractical at a transit instrument.

Conclusions. The null result of our blind survey is compatible with our present understanding of the Galactic population of millisecond pulsars. In particular, there does not seem to be a large population of fast-rotating millisecond pulsars.
\end{abstract}

Key words. methods: data analysis - galaxies: star clusters: general - pulsars: general - techniques: radar astronomy

\section{Introduction}

In the past fifteen years the timing of millisecond pulsars in the radio domain has become an important field of research for pulsars and their emission mechanisms. Important results have been obtained in neutron-star physics and General Relativity (see Lattimer \& Prakash 2007; and Lorimer 2008 for reviews). It has also been shown that, for certain pulsars, the stability of the rotation period is close to that of atomic time scales for an averaging time of several years (e.g. Janssen et al. 2010). However, this property generally does not hold when the observations extend over decades. Nevertheless, assuming a good stability of the rotation, a "pulsar" time scale may be calculated from a pulsar timing array (Taylor 1991; Petit \& Tavella 1996; Guinot \& Arias 2005; Manchester 2011).

It is thus essential to discover new millisecond pulsars in order to improve on the present results. In addition to better statistics on a larger set of data, one can hope to discover pulsars with a better stability of rotation. Presently, it seems that old millisecond pulsars with short period (less than $15 \mathrm{~ms}$ ) and thus small period derivative are the most plausible candidates for a stable period. Furthermore, the timing of a widely distributed sample of millisecond pulsars has the potential to detect gravitational waves at nanohertz frequencies (van Haasteren et al. 2009; Verbiest et al. 2009; Manchester 2011). Finally, the discovery of submillisecond pulsars would revolutionize fundamental physics, because the stiffest equations of state would then have to be abandoned (Haensel et al. 1995).

It is likely that only a small fraction of the Galactic population of millisecond pulsars has so far been discovered. One reason for a low detection rate is that the sensitivity of many surveys decreases strongly with decreasing rotation period, mainly for high values of interstellar dispersion, because of insufficient sampling resolution in time and frequency. In particular, the searches at the turn of the century for submillisecond pulsars were unsuccessful (D'Amico 2000; Crawford et al. 2000; Edwards et al. 2001).

This paper reports on a blind survey in the Galactic plane and three successive targeted searches conducted at the large radiotelescope of Nançay Observatory for short-period millisecond pulsars (ranging from 0.65 to $30 \mathrm{~ms}$ ). The project was initiated in the 1990 s to search for short-period pulsars with a technique 
of soft-correlation of radio signals developed for other applications. At the time, the recording of radio signals at a very high sampling rate and their subsequent treatment by soft-correlation techniques was quite a challenge in view of the performances of standard computers, and was only being done in VLBI with dedicated instruments. The first years were devoted to writing software to read the VHS (and later SVHS) video tapes on which the data were initially stored and to developing dedicated parallel computers based on transputers, and later on shark processors, to perform the actual soft-correlations, along with the necessary software. The blind survey started in 1997, a time when past surveys had searched only a fraction of the parameter space, because of the limited sampling resolution in time and frequency. It was designed to be complementary to past and ongoing surveys by only searching with high sampling resolution. When the blind survey proved less successful than expected, targeted searches were meant to provide additional chances of finding pulsars with limited additional observing time.

The acquisition method is explained in Sect. 2, the data processing in Sect. 3, and its validation in Sect. 4. The blind survey and the three targeted searches are presented in Sect. 5. We put the null result of the blind survey in perspective by estimating in Sect. 6 the chances of detecting short-period pulsars with our setup, by way of simulations on a model for the Galactic population of millisecond pulsars.

\section{Method of acquisition}

The observations were obtained at the large radiotelescope ${ }^{1}$ of Nançay Observatory. This is a transit instrument with a beam of $3.6 \times 22$ arcmin at zero declination and $1400 \mathrm{MHz}$.

In the initial stages of the project (1997-98), the data acquisition was done with the VLBI S2 system and SVHS video tapes. The signal from the receiver in two bands (1400 and $1410 \mathrm{MHz}$ ) and in both polarizations was registered, totalling four channels, each $6.4 \mathrm{MHz}$ wide. The sensitivity of the system was determined from the mean flux density of a detectable pulsar (7 sigmas). We measured a sensitivity of $3.5 \mathrm{mJy}$ for $240 \mathrm{~s}$ of integration, from the signal-to-noise ratio (SNR) of PSR B1937+21 (Rougeaux et al. 2000). We obtained 186 positions in the sky (hereafter points) of the blind survey with this setup. Shortly thereafter, the radiotelescope closed for renovation of the receiver, and the French space agency discontinued its support for this project, thus bringing observations and technical developments to a temporary halt.

In 2004 we replaced this system with a custom-made acquisition board that digitized the signal on one bit before sending it to an electronic board ADLink PCI-7300A revB, which stored the data on the $120 \mathrm{~Gb}$ hard disk of a PC running under Windows $2000 \mathrm{OS}$. The system sampled the signal at a rate of $50 \mathrm{Mb} / \mathrm{s}$ on one bit and a 24-MHz band, one polarization, one channel, at the frequency $1370 \mathrm{MHz}$. One channel of this system $(24 \mathrm{MHz})$ was equivalent to the old one $(4 \times 6.4 \mathrm{MHz})$, but the new system was much simpler to operate. We also confirmed the gain in sensitivity by a factor 2.2 of the new receiver of the radiotelescope after 2000. Procedures allowed the automatic acquisition of the data and their analysis in slightly differed time on PCs. The computation of the autocorrelation function with small time samples (typically $32 \mu \mathrm{s}$ ) was done on that computer before further treatment on more powerful computers (part of a cluster of 98 PC boards, and later two fast dual-core computers operating

\footnotetext{
1 http://www.obs-nancay.fr/en/index.php/instruments/ radio-telescope
}

under Linux OS). The integration time of a single pointing was set to $200 \mathrm{~s}$ and the corresponding sensitivity was $2.2 \mathrm{mJy}$. We obtained 2829 points of the blind survey with this setup.

In July 2006, a new custom-made acquisition board was installed at the radiotelescope. The signal was thereafter obtained at $1392 \mathrm{MHz}$, with a 48-MHz bandwidth and sampled at a rate of $100 \mathrm{Mb} / \mathrm{s}$, which raised the sensitivity by a factor 1.4 . The integration time of each pointing was reduced to $160 \mathrm{~s}$, so the sensitivity was $1.7 \mathrm{mJy}$. All three targeted searches and 2542 points of the blind survey were done with this setup.

The functioning of the backend was regularly checked by observations of PSR B1937+21, about twice a month.

\section{Data processing}

Our data processing scheme for 1-bit data has been described earlier (Rougeaux 1999; Rougeaux et al. 2000). Here we recall the algorithm used to detect pulsar signal in a single observation (Sect. 3.1). This processing method is hereafter called "survey mode". We then present the algorithm used to increase the sensitivity by using multiple consecutive observations (Sect. 3.2), which we call "cluster mode". The blind survey was reduced with the first method, while the reobservations of pulsar candidates in the blind survey and the three targeted searches were reduced with the other one.

\subsection{Processing in "survey mode"}

The first step of the processing is coherent folding of the signal. Then a search in a 3-dimensional parameter space (period $P$, dispersion slope $D$, and acceleration parameter $A$ ) is performed. The range of trial periods was initially $0.65-3.0 \mathrm{~ms}$. The lower limit corresponds to the fastest theoretically possible rotation period for a uniformly rotating neutron star (Haensel et al. 1995). Following the recommendation of the radiotelescope time allocation committee, we extended this range to $0.65-30 \mathrm{~ms}$ when we started the targeted searches at the end of 2006, at a low computational cost (about 25\%). We then also applied the extended range to the blind survey, thanks to the increased computational power available.

For a given set of trial values $P, D, A$, the folding process yields an SNR value $S(P, D, A)$. The typical numbers of trials are $N_{P} \simeq 10^{6}-2 \times 10^{6}$ for period, $N_{D} \simeq 100-200$ for dispersion slope, $N_{A}<10$ for acceleration. In addition, for each trial, the signal is searched for in about ten positions in phase so that the search space has about $10^{10}$ elements. All SNR values higher than a given threshold (e.g. for an SNR threshold of 4.0, typically $10^{6}$ elements out of $10^{10}$ ) are kept for further analysis. Because the acceleration parameters are relatively few, the results are examined independently for each value of this parameter. The retained values $S_{A}(P, D)$ are then grouped by peaks (clusters of adjacent points in the plane $P-D$ ).

Once this basic treatment is done, all signals with maximum SNR higher than some threshold (typically 6.5) are extracted and examined. Except when radio-interferences are present, the processing typically yields one such candidate peak every three to five observations. For the candidate peaks, the raw data are reanalyzed with slightly different trial values, centered on the candidate values, and with finer steps in period, dispersion slope and acceleration. Candidates that maintain the SNR for varying trial values (about one per 50-100 observations) are reobserved. 
Table 1. Known pulsars observed for checking the sensitivity.

\begin{tabular}{lcccccc}
\hline \hline Pulsar & $\begin{array}{c}P \\
\mathrm{~ms}\end{array}$ & $\begin{array}{c}D M \\
\mathrm{pc} \mathrm{cm}^{-3}\end{array}$ & $\begin{array}{c}\text { Flux } \\
\mathrm{mJy}\end{array}$ & $N$ & Obs SNR & $\begin{array}{c}\text { Obs flux } \\
\mathrm{mJy}\end{array}$ \\
\hline PSR B1620-26 & 11.076 & 63 & 1.6 & 4 & 19 & $1.9-2.1$ \\
PSR B1744-24A & 1.396 & 235 & 2.5 & 3 & $30-35$ & $3.0-3.5$ \\
PSR J1807-2459A & 3.059 & 134 & 1.1 & 3 & 8.4 & $0.8-0.9$ \\
PSR B1821-24 & 3.054 & 120 & 1.1 & 4 & 8 & $0.8-0.9$ \\
PSR J2051-0827 & 4.509 & 21 & 2.8 & 4 & 9 & $0.9-1.0$ \\
\hline
\end{tabular}

\subsection{Processing in "cluster mode"}

For $N_{\mathrm{O}}$ consecutive observations, the results are then $N_{\mathrm{O}}$ series $S_{i}(P, D, A)$, with $i$ from 1 to $N_{\mathrm{O}}$. For a pulsar in a binary system, $P$ may vary within each observation (i.e. the acceleration parameter is not zero), and $P$ will also vary between each observation, therefore a new parameter $P_{d}=P(i+1)-P(i)$ is needed. Obviously the parameters $A_{i}$ and $P_{d}$ are correlated. However, the $A_{i}$ are poorly determined in each observation, thus they cannot directly be used to connect candidate peaks between the $N_{\mathrm{O}}$ consecutive observations, especially as the candidate peaks are quite numerous (all SNR greater than typically 4-4.5). On the other hand, the dispersion measure of a pulsar is the same in all observations. The processing is then broken into $N_{D}$ independent procedures, each concerning one trial dispersion slope, for which the matching of $\left(P, A, P_{d}\right)$ is performed as follows.

The Hough transformation (Aulbert 2007) is used to transform the search in the plane $(T, P)$, where $T$ represents time (i.e. the sequence of observations), into one in the plane $\left(P_{d}, P\right)$. Assuming that the total time span is short enough for a pulsar period to vary linearly with time, in the first parameter space, a pulsar is represented by a line $P=P 0_{d} i+P 0$ where $P 0$ is the period at the reference epoch, and $P 0_{d}$ the period derivative. The line representing a pulsar is to be found as one that fits the measurement results $\left(i, P_{i}\right)$. In the second parameter space $\left(P_{d}, P\right)$, the measurement results are transformed to lines $P=P_{i}-i P_{d}$ and the pulsar is represented by a single point $\left(\mathrm{PO}_{d}, P 0\right)$. The point representing the pulsar is found at an intersection of lines.

With respect to this basic procedure (see a more detailed description in Aulbert 2007), two additional features are introduced, as each measurement $\left(i, P_{i}\right)$ comes with an SNR $S_{i}$ and an acceleration parameter $A_{i}$. First, the acceleration parameter is used to constrain the $P_{d}$ value; i.e., each measurement produces not a full line, but a segment in the $\left(P, P_{d}\right)$ plane. Second, each segment is assigned $S_{i}$ as a weight so that each intersection of lines comes with the combined weight of the participating segments, in effect providing the detection SNR if the intersection of lines corresponds to a real pulsar.

The results $S\left(P 0, P 0_{d}\right)$ for all dispersion slopes $D$ are then assembled and grouped by peaks (clusters of adjacent points in the plane $P-D)$ and peaks with large SNR are examined. The couple $\left(P 0, P 0_{d}\right)$ for the maximum SNR provides the period and period derivative of the observed, or candidate, pulsar. An example of such a processing is given in Sect. 4.2.

\section{Theoretical performance}

The point source efficiency at zero declination is about $1.6 \mathrm{~K} / \mathrm{Jy}$ at $1400 \mathrm{MHz}$, and the system temperature is about $35 \mathrm{~K}$, which leads to a 7-sigma theoretical detection threshold of $1.2 \mathrm{mJy}$ for one 160 -s observation at $100 \mathrm{Mb} / \mathrm{s}$. The actual sensitivity is lower for several reasons: loss due to the 1-bit digitization, decrease in sensitivity with declination differing from 0 , losses due to rounding in the folding process, etc.
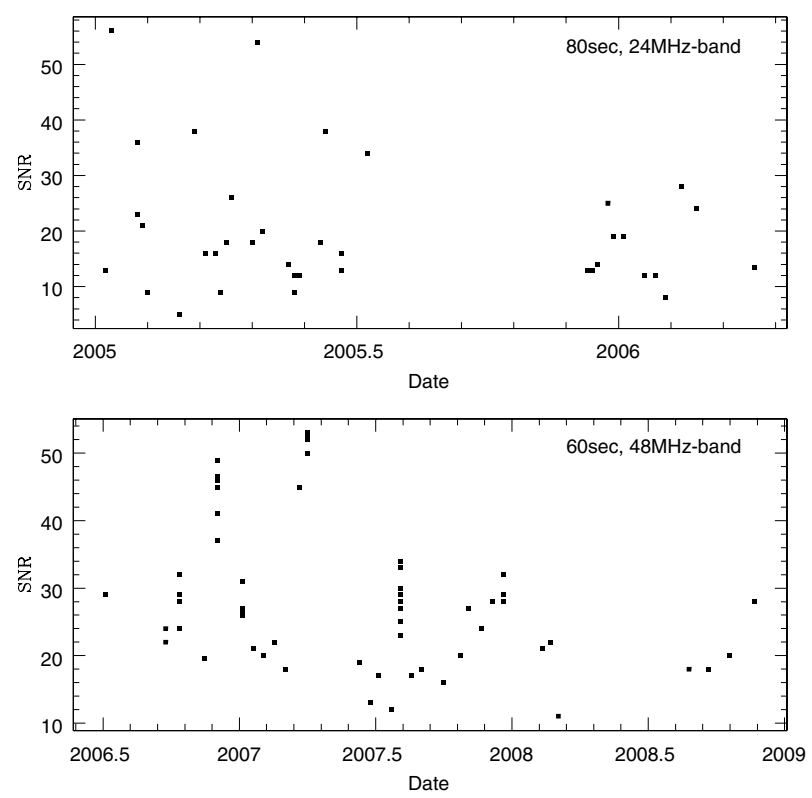

Fig. 1. Signal-to-noise ratio for single observations of PSR B1937+21 and reduction in "survey mode". Top: 80 -s pointings with second setup (24-MHz bandwidth). There were no observations in the second semester of 2005 because of a backend malfunction. Bottom: 60 -s pointings with third setup (48-MHz bandwidth). These regular observations of the pulsar were made to check the functioning of the backend and monitor the sensitivity of the setup.

The sensitivity of the targeted search was tested by the detection of known faint pulsars in globular clusters, in the framework of the targeted search in globular clusters (see Sect. 5.2). Table 1 gives the results for five pulsars, each observed with three or four successive integrations of $160 \mathrm{~s}$ (see more details in Sect. 4.2).

\subsection{Performance validation for "survey mode"}

We regularly observed the millisecond pulsar PSR B1937+21 $\left(P=1.557 \mathrm{~ms}, D M=71 \mathrm{~cm}^{-3} \mathrm{pc}\right)^{2}$ in order to check the operation of the system and to obtain estimates of the achieved detection sensitivity. With the last setup, we obtained 99 60-s observations on 32 different days (see Fig. 1). The average detection SNR is 28.3 and the median value is 27 .

Our results imply that PSR B1937+21 would be detected with an SNR of 46 (or 44 when using the median value) with an integration of $160 \mathrm{~s}$. Assuming that the main pulse of PSR B1937+21 is a source with a mean flux density of $10 \mathrm{mJy}$, we infer a 7-sigma detection threshold of $1.52 \mathrm{mJy}$ (or $1.59 \mathrm{mJy}$ when using the median). Taking a safety margin, we can state

2 Most data on known pulsars were taken from the ATNF pulsar catalog (http://www.atnf.csiro.au/research/pulsar/psrcat/); see also Manchester et al. (2005). 

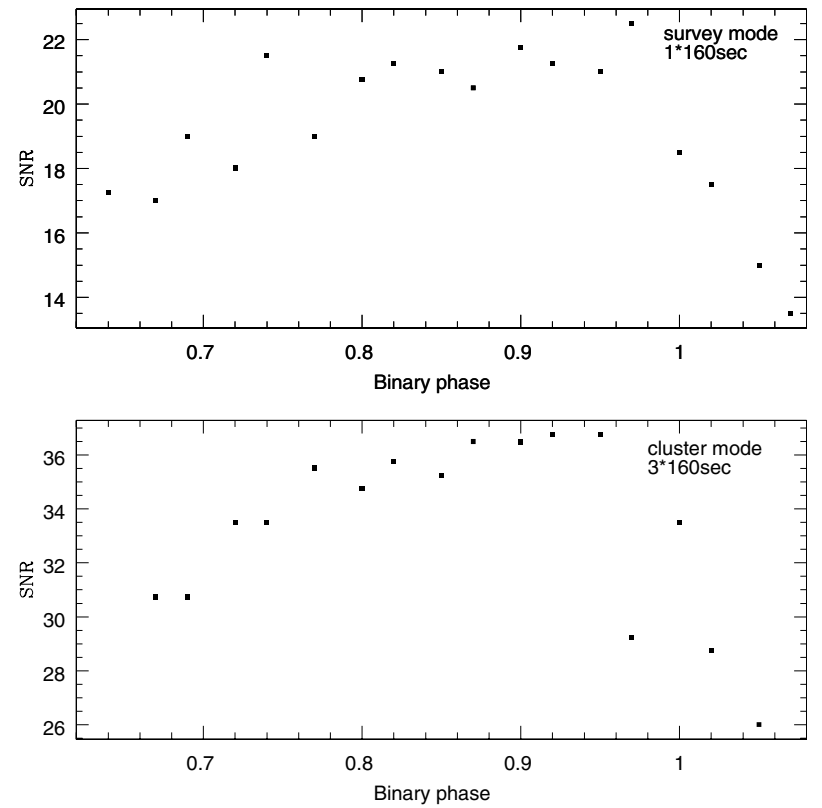

Fig. 2. Signal-to-noise ratio for observations of PSR B1744-24A with third setup. Top: single 160-s integrations and reduction in "survey mode". Bottom: three 160-s integrations and reduction in "cluster mode".

that the sensitivity of the blind survey is about $1.7 \mathrm{mJy}$ for one 160-s integration with the backend installed in July 2006.

\subsection{Performance validation for "cluster mode"}

We have validated the strategy for processing consecutive observations of an accelerated pulsar by applying it to the binary pulsar PSR B1744-24A in the globular cluster Terzan 5. This pulsar is strong enough (when not in eclipse) to be easily detected and is in a close enough binary system to display relatively large variations in the period and period derivative within typical observing times (a few 160-s observations). We carried out 18 consecutive 160-s observations on 16 March 2007.

The 18 observations were then processed in 16 groups of three observations, following the procedure described in Sect. 3.2. The detection SNR values obtained in these analyses are shown in Fig. 2. They are perfectly consistent with the coherent addition of the SNR values obtained by treating all integration separately, except for one point at binary phase 0.98 for which the assumption that the pulsar period varies linearly does not hold sufficiently over three consecutive observations.

In our search for new pulsars, we also observed globular clusters with known pulsars, which allowed us to check the processing in "cluster mode" (the same validation can also be performed on field pulsars, but at the cost of some extra observing time). Table 1 presents a summary of the results that we now explain in some more details. As an example, PSR J1807-2459A in the cluster NGC 6544 has an expected flux density of $1.1 \mathrm{mJy}$ at $1400 \mathrm{MHz}$, thus would yield an SNR that is quite insufficient for detection using only one 160-s integration. Processing of three consecutive observations yields an SNR of 8.4, allowing detection in a wide search. Similar detections were obtained for several other pulsars. For PSR B1821-24 in NGC 6626, the flux density is $0.18 \mathrm{mJy}$ in ATNF, but $1.1 \mathrm{mJy}$ in Lyne et al. (1987). We obtained an SNR of 8 for four successive 160-s observations, which seems to favor the second value. For PSR B162026 in NGC 6121, with an expected flux density of $1.6 \mathrm{mJy}$, we

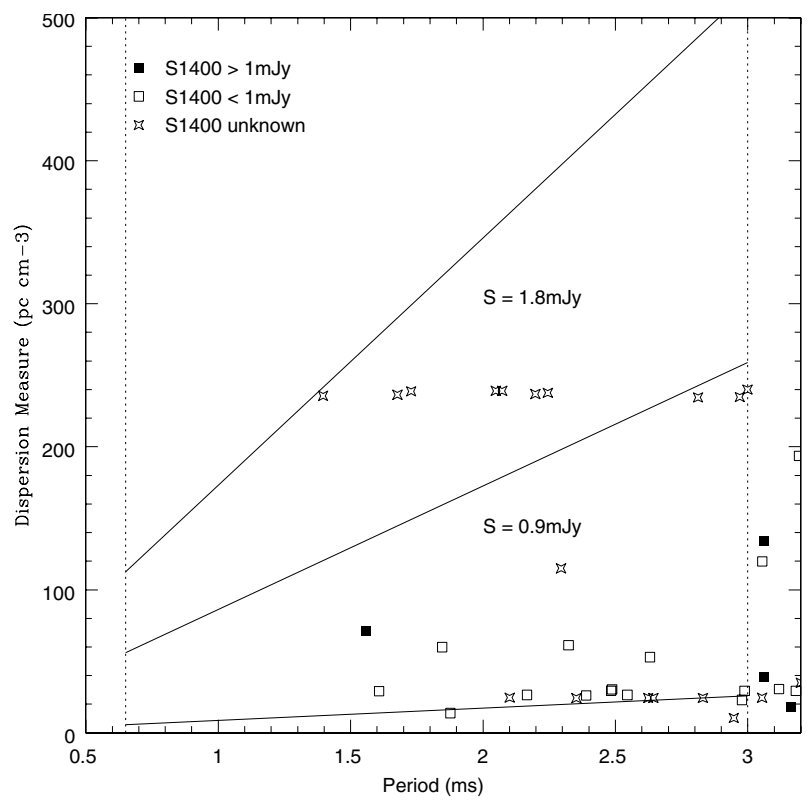

Fig. 3. Dependence of the sensitivity on dispersion measure. The sensitivity decreases progressively toward higher dispersion measures. The diagonal lines correspond to a dispersion representing $0.1,1$, and 2 windows per frequency channel, respectively. The individual points are known millisecond pulsars with different symbols depending on their flux density at $1400 \mathrm{MHz}$.

obtained an SNR of 19 for four 160-s observations. For the field eclipsing pulsar PSR J2051-0827, the expected flux density is quite variable and is reported at $2.8 \mathrm{mJy}$ in ATNF, but only $1.1 \mathrm{mJy}$ in Stappers et al. (1996). We obtained an SNR of 9 for four 160-s observations, which is consistent with the second value. Overall, we can expect to obtain a 8-sigma detection threshold at about $1 \mathrm{mJy}$ or slightly below with four consecutive observations.

\subsection{Dependence of the actual sensitivity on dispersion measure}

The sensitivities quoted above are nominal sensitivities, and the actual ones will depend on the dispersion measure at the distance of the pulsar. This is true for any processing method.

In our method, the power spectrum is computed over time windows of a small fraction of the tested period and typically uses 128 frequency channels. Because of the 1-bit sampling the spectrum is normalized and we rely on the variation in the power (with time across the windows and with frequency across the channels) due to the dispersion of the pulse to detect a pulsar. The effect of the dispersion measure on the sensitivity is shown in Fig. 3. Starting from the bottom, the first diagonal line is the limit below which we can hardly detect pulsars because the dispersion is too low (less than 0.1 window per frequency channel), and the signal is spread equally in all frequency channels. As the dispersion increases, the signal for a given window in time is spread over fewer frequency channels, and contrast appears between channels with and without signal. The sensitivity is optimal (i.e. $0.9 \mathrm{mJy}$ for four integrations of $160 \mathrm{~s}$ and reduction in "cluster mode") in the region between the first and second diagonal line, i.e., between 0.1 and 1 window per frequency channel. Above the second diagonal line, the sensitivity decreases by a factor 2 because the dispersion is between one and two windows per frequency channel. Above the top diagonal line, the 
sensitivity loss is such that we simply did not search that part of the parameter space during processing. These are not strict limits, however, at neither the low nor the high end. For example, the signal of the pulsar is spread over 20 channels out of 128 for a dispersion of 0.05 window per frequency channel, so there is still some contrast in the power spectrum between a window with no signal and a window with a signal in some part of the band. For high dispersion values, the pulse is spread over several windows so the fraction of the signal in each window is reduced, and furthermore the contrast in the power spectrum of a given window is also reduced, because the signal will appear in different channels for different pulses.

There are other practical reasons for limiting the search to the interval between 0.1 and 2 windows per frequency channel. Below 0.1, pulsars with such low dispersion must be nearby and should already have been detected, and above 2, pulsars with such high dispersion measure are more likely to be distant, thus too faint given the decreased sensitivity.

\section{The searches}

Our initial goal was to do a blind survey in the Galactic plane. But after two years of unsuccessful searching, we estimated that the probability of return per hour of observing time was probably higher in targeted searches than in a blind survey, so we decided to try them as well.

There are several possible types of targeted searches for millisecond pulsars. The most successful by far is to point at Galactic globular clusters. This is where most recent searches for such objects have been done (e.g. Possenti et al. 2003; Ransom et al. 2005; Freire et al. 2005). Another option is to select radiosources with no optical counterpart in catalogs of radiosources, as done by several groups (Crawford et al. 2000; Han et al. 2004), and with success by Navarro et al. (1995). Pulsars can be distinguished from other radiosources in blind surveys by their steep spectral index and by a high degree of polarization, usually above 5\%. Millisecond pulsars tend to show the same, sometimes high, degree of linear polarization as ordinary pulsars (Yan et al. 2011).

\subsection{The blind survey}

The blind survey was conducted in the Galactic plane, where most pulsars are likely to be: latitude $(b)$ smaller than $3^{\circ}$, longitude $(l)$ between $20^{\circ}$ and $110^{\circ}$. One could argue that in setting a limit in $b$ rather than in height above (or below) the Galactic plane, we miss nearby pulsars that are at high $b$ and in the Galactic plane. However, as shown below (Sect. 6.2), the gain in detectable pulsars would be largely offset by the cost in telescope time.

A typical observing run was done at a set declination and lasted 20 to $120 \mathrm{~min}$ (depending on the declination and the alloted time), shifting the telescope (in fact the receiver, since this is a transit instrument) by one beam in right ascension after each short integration. A better observing strategy was to shift by multiples of one beam and return to that declination at later dates to fill the gaps. The selected declinations were also separated by one beam, so that all pointings were separated by at least one beam in either direction.

The total amount of telescope time attributed to this project over the years was $549.3 \mathrm{~h}$, at which point the time allocation committee wisely decided to cut its losses. We observed 186 points in the sky with the first setup (sensitivity $\simeq 3.5 \mathrm{mJy}$ for

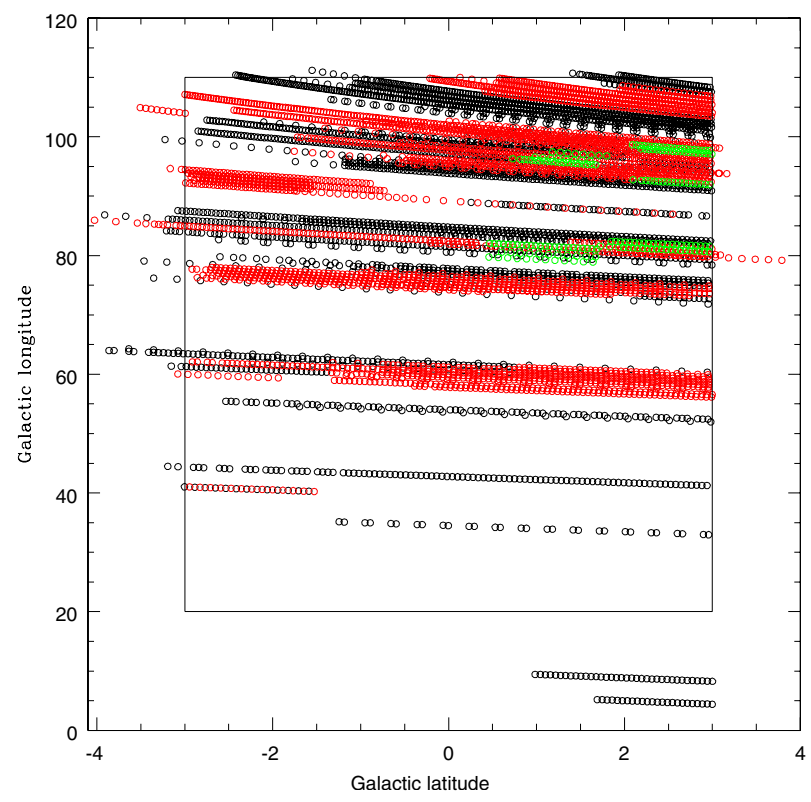

Fig. 4. Map of the observed points in the blind survey in the Galactic plane. The green, red, and black open circles correspond to observations with the first, second, and third setups, respectively. The size of the symbols is constant and not representative of the radiotelescope beam, which is elongated in declination and increases with declination (thus roughly with longitude). The inner rectangle encloses the region of the nominal survey. Regions below $l=70^{\circ}$ are poorly covered because of oversubscription of the radiotelescope time at lower longitudes.

integrations of $240 \mathrm{~s}$ ), 2829 points with the second setup (sensitivity $\simeq 2.2 \mathrm{mJy}$ for integrations of $200 \mathrm{~s}$ ) and 2542 points with the last setup (sensitivity $\simeq 1.7 \mathrm{mJy}$ for integrations of $160 \mathrm{~s}$ ). Seventy points were observed several times, leading to a total of 5487 different points observed out of a total of 22057 , that is, a coverage of $25 \%$ of the planned survey. The actual sensitivity was different in some of the pointings. In the second semester of 2004, the sensitivity progressively degraded from 2.2 to $2.7 \mathrm{mJy}$ due to an unnoticed desynchronization in the acquisition. A bug in the treatment software in late December 2004 lowered the sensitivity by about half for 57 points. We attributed a slightly higher sensitivity (by an arbitrary amount of $20 \%$ ) to the 70 points observed repeatedly, because of the better chance of detecting pulses as the effective flux may vary and as the fixed set of tested values in the parameter space may better match the actual parameters of the pulsar.

A map of the survey region in Galactic coordinates with the covered region shown as circles (colors depending on setup) is presented in Fig. 4. The figure shows that a few points were observed outside the region of the survey, either by mistake, or by opportunity (to save repointing the telescope). The figure also shows that regions below $l=70^{\circ}$ are poorly sampled, because of oversubscription of the telescope time by a factor greater than 2 at the corresponding right ascensions. While millisecond pulsars are likely to be more frequent at lower Galactic longitudes (closer to the Galactic center), their a priori detectability is strongly reduced by pulse smearing at longitudes below $50^{\circ}$ (see Sect. 6 and Fig. 5). However, the drawback of higher declinations (and thus higher longitudes) is that the antenna aperture efficiency is progressively reduced, with a loss of sensitivity of $15 \%$ at $l \simeq 85^{\circ}$ and $28 \%$ at $l \simeq 110^{\circ}$ (Fouqué et al. 1990). About half of our pointings suffer a loss of sensitivity of $20 \%$ or more because of that effect. 

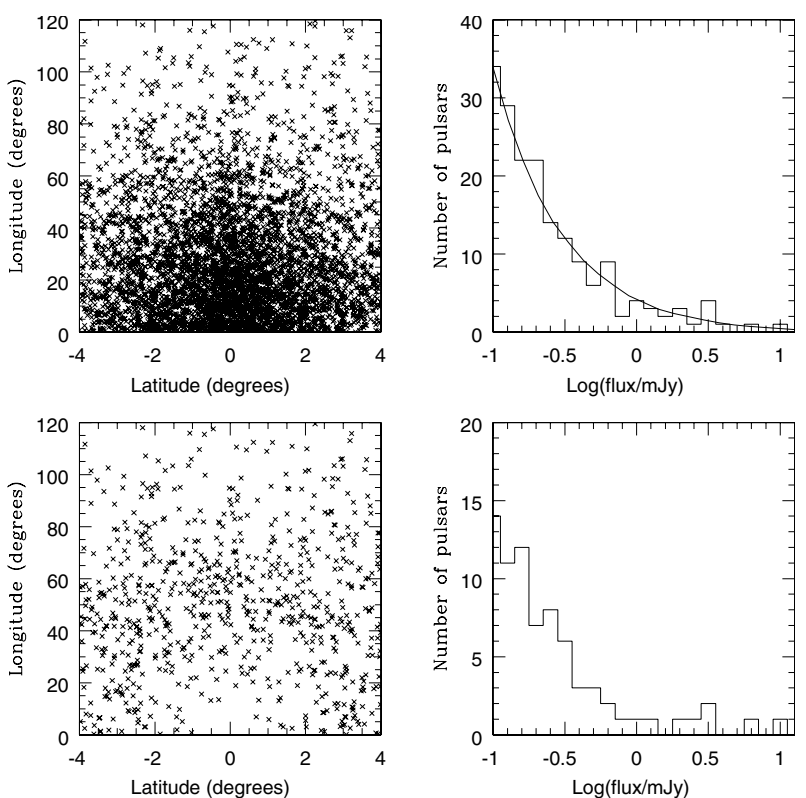

Fig. 5. One simulation of the population of 30000 millisecond pulsars in the Galaxy. Top: distribution of all (4589) short-period pulsars in the region of the blind survey and corresponding histogram of flux densities; the solid curve is the mean histogram from 1000 simulations. Bottom: distribution of the (730) pulsars remaining after eliminating the undetectable ones (because of pulse smearing at high dispersion measure) and corresponding histogram of flux densities. Both histograms are limited to flux densities higher than $0.1 \mathrm{mJy}$ for clarity.

During the survey we identified 25 pulsar candidates with an $\mathrm{SNR} \simeq 7$. They are listed in Table 2 , together with their coordinates (1950 equinox), probable period (in $\mathrm{ms}$ ) and the number of 160-s integrations in the reobservations. Note that the last two candidates were not reobserved for lack of observing time. Their reobservation and analysis in "cluster mode" (see Sect. 3.2) did not enable us to confirm them. Some of these presumably false detections can be explained by interferences from radars at civilian and military airports near Paris.

\subsection{Globular clusters}

The central regions of globular clusters are favorite targets when searching for millisecond pulsars, because they are regions of very high stellar density. In such regions, multiple encounters between stars favor the formation of binary stars (Bailyn 1995), and, when one of the stars is a pulsar, favors the acceleration of the pulsar to periods of about a millisecond (pulsar recycling). Furthermore, a large number of binary stars in globular clusters may in fact be primordial binaries (Hut et al. 1992), thus increasing the probability of finding recycled pulsars in globular clusters.

We selected globular clusters as targets using three criteria:

- avoid the zone accessible to the radiotelescope in Arecibo, which, with its large collecting area and its new ALFA receiver, can conduct much more sensitive searches than us;

- aim at nearby globular clusters, with high stellar density, and where no millisecond pulsars have been detected so far;

- select preferentially those clusters where the dispersion measure is highest, and hence where pulsars are difficult to detect with some of the search techniques used in past surveys (see below).
Table 2. List of pulsar candidates with SNR $>6.5$.

\begin{tabular}{ccccc}
\hline \hline Name & RA(B1950) & Dec(B1950) & $\begin{array}{c}P \\
\mathrm{~ms}\end{array}$ & $N$ \\
\hline 4091907 & 200025.36 & +25100 & 0.876 & 10 \\
2541105 & 201018.22 & +37160 & 0.885 & 4 \\
2271416 & 201800.63 & +37160 & 1.071 & 4 \\
4830818 & 202916.4 & +43520 & 1.047 & 7 \\
3690234 & 202922.06 & +38220 & 1.171 & 11 \\
5191628 & 203044.2 & +40560 & 0.723 & 3 \\
5141841 & 203354.2 & +40560 & 4.347 & 3 \\
4481402 & 203556.31 & +41400 & 0.892 & 4 \\
3760145 & 204119.79 & +38440 & 1.742 & 10 \\
4942356 & 204151.00 & +43520 & 0.997 & 5 \\
4780947 & 204830.47 & +43520 & 3.579 & 8 \\
4561319 & 205408.7 & +41400 & 2.628 & 4 \\
& & & 0.920 & \\
4721049 & 211353.4 & +43080 & 2.009 & 4 \\
3630610 & 213602.95 & +51560 & 0.954 & 4 \\
3680335 & 214102.86 & +54520 & 1.738 & 4 \\
3161822 & 220225.0 & +58540 & 2.747 & 4 \\
2791002 & 220740.95 & +55360 & 1.720 & 4 \\
3072105 & 220850.2 & +58320 & 1.783 & 4 \\
4251900 & 221055.6 & +54080 & 0.675 & 10 \\
4012312 & 221651.3 & +54080 & 0.952 & 7 \\
3092112 & 223251.15 & +58320 & 1.425 & 4 \\
4032330 & 224230.55 & +61280 & 1.248 & 7 \\
2210226 & 224920.78 & +60220 & 2.108 & 4 \\
2140142 & 201512.55 & +36320 & 2.489 & $a$ \\
3451149 & 220912.42 & +54300 & 1.492 & $a$ \\
\hline
\end{tabular}

Notes. ${ }^{(a)}$ Not reobserved for lack of observing time.

To test the feasability of our project, we selected in the ATNF the 32 pulsars discovered in globular clusters as of April 2006 with a published flux density. If the flux density was given at $400 \mathrm{MHz}$, it was converted to $1400 \mathrm{MHz}$ assuming a spectral index of -1.8 . We then calculated the number $N_{\mathrm{p}}$ of these 32 pulsars that would be detected if they were all in each of the different Galactic globular clusters. We selected those clusters in which we have a reasonable chance of detected millisecond pulsars taking $N_{\mathrm{p}}$, the sensitivity of our search, and the value of the dispersion measure at the distance of the cluster into account. Other clusters were added in the course of the search to test the sensitivity for reduction in "cluster mode" (see Sect. 4.2) or to optimize the use of the available telescope time.

We obtained $28.33 \mathrm{~h}$ ( +3 of discretionary director's time) of telescope time for this project. The list of observed globular clusters and their characteristics are given in Table 3. The successive columns are the name of the cluster, the range in dispersion measure (from the model of Cordes \& Lazio 2002), or the dispersion measure (from the ATNF catalog) if millisecond pulsars have been detected in the cluster, in $\mathrm{cm}^{-3} \mathrm{pc}$, the expected number $N_{\mathrm{p}}$ of pulsars with a flux density above $1 \mathrm{mJy}$ (assuming that all 32 know millisecond pulsars are in that cluster), the date of observation, and the number of pointings.

Candidates were detected in the clusters NGC 6171, NGC 6254 and NGC 6809, which were reobserved repeatedly. No new millisecond pulsar was detected.

\subsection{Bright polarized point-like radiosources with steep spectra}

In the second targeted search, the candidate pulsars were selected from the catalog of steep spectral index radiosources 
Table 3. List of the observed globular clusters.

\begin{tabular}{|c|c|c|c|c|c|c|c|c|c|}
\hline Cluster & $\begin{array}{c}D M \\
\text { pc } \mathrm{cm}^{-3}\end{array}$ & $N_{\mathrm{p}}$ & $\begin{array}{c}\text { Date } \\
\text { yyyymmdd }\end{array}$ & $N$ & Cluster & $\begin{array}{c}D M \\
\text { pc } \mathrm{cm}^{-3}\end{array}$ & $N_{\mathrm{p}}$ & $\begin{array}{c}\text { Date } \\
\text { yyyymmdd }\end{array}$ & $N$ \\
\hline NGC 5986 & $169-171$ & 0 & 20070213 & 4 & & & & 20070815 & 18 \\
\hline NGC 6093 & $108-109$ & 0 & 20070312 & 4 & & & & 20070816 & 18 \\
\hline NGC 6121 & 63 & 20 & 20070213 & 4 & NGC 6553 & $237-350$ & 4 & 20070313 & 4 \\
\hline \multirow[t]{5}{*}{ NGC 6171} & $83-86$ & 4 & 20061119 & 3 & & & & 20071211 & 8 \\
\hline & & & 20070213 & 4 & NGC 6624 & 87 & 0 & 20070427 & 4 \\
\hline & & & 20070426 & 4 & NGC 6626 & 121 & 5 & 20070211 & 4 \\
\hline & & & 20071005 & 10 & NGC 6712 & $235-333$ & 3 & 20061114 & 3 \\
\hline & & & 20071201 & 10 & & & & 20070313 & 4 \\
\hline \multirow[t]{2}{*}{ NGC 6218} & $70-75$ & 7 & 20061119 & 3 & & & & 20071221 & 18 \\
\hline & & & 20070213 & 4 & NGC 6717 & $163-193$ & 2 & 20061114 & 3 \\
\hline \multirow[t]{6}{*}{ NGC 6254} & $73-81$ & 8 & 20061119 & 3 & & & & 20070923 & 18 \\
\hline & & & 20070312 & 4 & NGC 6760 & $208-310$ & 1 & 20061203 & 4 \\
\hline & & & 20070426 & 4 & NGC 6809 & $103-113$ & 6 & 20061203 & 4 \\
\hline & & & 20070624 & 18 & & & & 20070117 & 4 \\
\hline & & & 20070718 & 18 & & & & 20070504 & 18 \\
\hline & & & 20071101 & 10 & & & & 20071121 & 10 \\
\hline NGC 6256 & $418-554$ & 0 & 20071201 & 10 & 2MASSGC01 & $144-292$ & 13 & 20070210 & 4 \\
\hline NGC 6266 & 115 & 3 & 20070313 & 4 & & & & 20070719 & 18 \\
\hline \multirow[t]{3}{*}{ NGC 6304} & $224-312$ & 4 & 20070119 & 4 & 2MASSGC02 & $127-175$ & 7 & 20070211 & 4 \\
\hline & & & 20070210 & 4 & & & & 20070922 & 10 \\
\hline & & & 20070815 & 18 & FSR1767 & $61-110$ & 25 & 20070922 & 8 \\
\hline NGC 6342 & 103 & 0 & 20070426 & 4 & Glimpse-CO2 & $294-560$ & 5 & 20080919 & 4 \\
\hline \multirow[t]{2}{*}{ NGC 6366} & $69-85$ & 13 & 20070119 & 4 & & & & 20081122 & 5 \\
\hline & & & 20070516 & 4 & IC 1276 & $176-251$ & 5 & 20070427 & 4 \\
\hline NGC 6440 & 224 & 0 & 20070427 & 4 & & & & 20071113 & 18 \\
\hline NGC 6441 & $366-451$ & 0 & 20070427 & 4 & Terzan10 & $268-413$ & 5 & 20071211 & 8 \\
\hline NGC 6544 & 134 & 16 & 20061114 & 3 & Terzan12 & $197-332$ & 7 & 20061114 & 3 \\
\hline \multirow[t]{2}{*}{ NGC 6540} & $212-315$ & 13 & 20070119 & 4 & & & & 20070211 & 4 \\
\hline & & & 20070516 & 18 & & & & 20071012 & 18 \\
\hline
\end{tabular}

published by De Breuck et al. (2000). While their catalog was constructed with the aim of obtaining a large sample of highredshift radiosources, because spectral index tends to increase with the redshift of optically identified sources, a large negative spectral index is also a property of millisecond pulsars.

We extracted the information on polarization and on source size from the NVSS ${ }^{3}$ catalog of radiosources. From this complete list, we extracted the sources that were unresolved and polarized $(\geq 5 \%)$. These criteria give 26 sources, two of which are in fact known pulsars (PSR J0630-2834 and PSR J2313+4253). We removed them from the list and checked that the 24 other sources are not in any database (Simbad, NED, Hyperleda). The final list is given in Table 4, together with their $1400-\mathrm{MHz}$ flux density in mJy, spectral index between 1400 and $326 \mathrm{MHz}$, percent polarization, the date of our observation, and the number of 160-s integrations. More information on the sources can be found in De Breuck et al. (2000).

We obtained $18.67 \mathrm{~h}$ of telescope time for this project and observed all the sources in the list. None of them turned out to be a pulsar.

\subsection{Faint polarized point-like radiosources}

In the third search, we extracted the candidate pulsars from the NVSS catalog. This catalog contained 97 of the 1775 pulsars in the ATNF catalog at the time of the project (early 2008). From the NVSS catalog, we first extracted the sources with $1400-\mathrm{MHz}$ flux densities between 2 and $10 \mathrm{mJy}$. We did not try to determine their spectral index, as in the previous set of targets, because
Table 4. List of observed bright polarized unidentified radiosources with steep spectrum.

\begin{tabular}{lccccc}
\hline \hline Name & $\begin{array}{c}\text { S1400 } \\
\text { mJy }\end{array}$ & $\alpha$ & $\%$ pol. & $\begin{array}{c}\text { Date } \\
\text { yyyymmdd }\end{array}$ & $N$ \\
\hline WN J0751+3300 & 10.7 & -1.47 & 5 & 20080107 & 18 \\
WN J0829+3834 & 10.9 & -1.49 & 5 & 20080202 & 18 \\
TN J0856-1510 & 68.5 & -1.41 & 12 & 20080304 & 18 \\
WN J0928+6003 & 10.4 & -1.63 & 7 & 20080402 & 18 \\
WN J1101+3520 & 14.9 & -1.32 & 5 & 20080204 & 18 \\
WN J1111+3311 & 12.3 & -1.32 & 6 & 20080319 & 18 \\
WN J1152+3732 & 14.7 & -2.18 & 5 & 20080110 & 18 \\
WN J1258+3212 & 18.3 & -1.52 & 8 & 20080306 & 18 \\
WN J1300+5311 & 18.1 & -1.44 & 5 & 20080214 & 18 \\
WN J1330+6505 & 9.5 & -1.34 & 5 & 20080413 & 3 \\
WN J1337+3401 & 13.2 & -1.39 & 7 & 20080116 & 12 \\
WN J1359+7446 & 11.2 & -1.92 & 5 & 20080413 & 4 \\
WN J1400+4348 & 18.2 & -1.49 & 6 & 20080205 & 4 \\
WN J1421+3103 & 11.6 & -1.43 & 5 & 20080413 & 3 \\
WN J1435+3523 & 20.8 & -1.35 & 7 & 20080320 & 12 \\
TN J1515-2651 & 56.7 & -1.34 & 9 & 20080218 & 18 \\
WN J1606+4142 & 10.9 & -1.31 & 10 & 20080407 & 18 \\
WN J1723+5822 & 10.1 & -1.46 & 11 & 20080111 & 8 \\
WN J1734+3606 & 7.1 & -1.31 & 14 & 20080117 & 8 \\
WN J1739+5309 & 9.8 & -1.32 & 5 & 20080111 & 8 \\
WN J1819+6213 & 11.6 & -1.45 & 7 & 20080123 & 12 \\
WN J1923+6047 & 11.8 & -1.30 & 8 & 20080419 & 18 \\
TN J2028-1934 & 131.4 & -1.34 & 7 & 20080302 & 15 \\
WN J2221+3800 & 13.2 & -1.32 & 5 & 20080422 & 18 \\
\hline
\end{tabular}

\footnotetext{
${ }^{3}$ http://www.cv.nrao.edu/nvss/
} 
there is no hope of finding these faint sources in catalogs at other frequencies.

We then selected those sources that were polarized (polarized flux density $>20 \%$ of total flux density) and with SNR in total and polarized flux density above 3. This produced a list of 2105 sources. In this list, we found six pulsars (PSR J0358+5413, PSR J0538+2817, PSR J0814+7429, PSR J0922+0638, PSR J1745-3040, PSR J1840+5640), none of which has a very short period. But one should note that none of the 45 known millisecond pulsars with periods less than $3.3 \mathrm{~ms}$ has $1400-\mathrm{MHz}$ flux densities above $2 \mathrm{mJy}$, except PSR B1937+21, so they cannot be in that catalog. We then further restricted the sample to the Galactic plane $\left(|b|<12^{\circ}\right)$, also eliminating the region $75^{\circ}<l<165^{\circ}$ and $|b|<5.5^{\circ}$ (recently surveyed by Hessels et al. 2007), the region of our own blind survey, and the right ascensions heavily oversubscribed at Nançay (17h00-19h40). We removed all sources identified in NED, SIMBAD, and 2MASX. We also carefully examined the postage-stamp images of all the sources and removed those that looked like noise, as well as close double sources (which are likely to be double-lobed radiogalaxies). This brought the total number of targets to 92. Some ambiguous cases (multiple sources, not quite point-like sources) were left in the sample, since at this low flux level it is sometimes difficult to distinguish real features from noise.

We were given $21.67 \mathrm{~h}$ of telescope time for this project, which enabled us to observe 65 of them (with one source observed twice). They are listed in Table 6, which gives the NVSS name, the flux density at $1400 \mathrm{MHz}$ in $\mathrm{mJy}$, and the percent polarization. This last targeted search did not produce any new millisecond pulsar, which seems to confirm that most radiosources with ultrahigh polarization are radio-loud active galactic nuclei (Shi et al. 2010).

The conclusion of the two targeted searches in catalogs of radiosources is that the luminosity function of millisecond pulsars is simply too faint to allow a meaningful number of them to be found in large surveys like NVSS or FIRST. The small number of sources observed does not allow us to be more quantitative.

\section{The Galactic population of millisecond pulsars}

We now examine whether the null result of our blind survey is statistically compatible with the Galactic distribution of millisecond pulsars as we know it. The method is the following. We adopt a model for the distribution of millisecond pulsars in space, rotation period, and luminosity, produce a large number of Galactic populations of synthetic millisecond pulsars, and, for each realization of the simulation, we compute how many synthetic pulsars should be detected in our blind survey, by comparing the properties of each synthetic pulsar (position, rotation period, flux density) with the coordinates and actual sensitivity of each of our 5487 pointings, taking the effect of the dispersion measure on the detectability into account in each case. The number of detected pulsars should be zero in most realizations, otherwise our model overestimates the true number of millisecond pulsars.

\subsection{Model for the Galactic population of pulsars}

Several models have been proposed over the years to characterize the population of pulsars in the Galaxy, either ordinary (Lyne et al. 1985; Yusifov \& Küçük 2004; Lorimer et al. 2006;
Faucher-Giguère \& Kaspi 2006), millisecond (Lorimer et al. 1996; Cordes \& Chernoff 1997; Story et al. 2007), or both (Lyne et al. 1998; Faucher-Giguère \& Loeb 2010).

The population of millisecond pulsars is too small to allow for a precise modeling of their spatial distribution. However, they are thought to have larger radial and vertical scale lengths than those of ordinary pulsars, because they are likely to have lost the memory of their birth location due to a high initial velocity.

We adopt the spatial distribution used by Faucher-Giguère \& Loeb (2010). The radial density in the Galactic plane is $\rho(r) \propto \exp \left(-r^{2} / 2 \sigma^{2}\right)$, where $r$ is the radial distance from the Galactic center and $\sigma=5 \mathrm{kpc}$. For ordinary pulsars, $\sigma=3.6 \mathrm{kpc}$ (Yusifov \& Küçük 2004). Our simulations and others (Lorimer et al. 1996) have shown that the exact radial dependence is in fact not important, because most millisecond pulsars are faint, and the heavily populated region toward the Galactic center is "obscured" by a high dispersion measure (see Fig. 5). Most detectable millisecond pulsars are thus within 2 or $3 \mathrm{kpc}$ of the Sun (which in this paper is assumed to be located $8 \mathrm{kpc}$ from the Galactic center). The distribution perpendicular to the Galactic plane is a simple exponential: $\rho(z) \propto \exp (-|z| / h)$, where $z$ is the distance from the Galactic plane and the scale height $h=500 \mathrm{pc}$ (Cordes \& Chernoff 1997; Story et al. 2007). This value is at the upper end of those estimated for ordinary pulsars (330-600 pc). Adopting a higher value for the scale height slightly reduces the number of pulsars at low Galactic latitudes, and hence the probability of detection in our blind survey. We do not take into account a possible increase in $h$ with $r$ (Yusifov 2004), which would reduce the number of pulsars in the Galactic plane even more.

The total number of millisecond pulsars is highly uncertain. Lorimer et al. (1995) estimate that there are 40000 millisecond and low-mass binary pulsars above $L_{\min }=2.5 \mathrm{mJy} \mathrm{kpc}^{2}$ at $436 \mathrm{MHz}$. Lyne et al. (1998) estimate that there are 30000 mil-

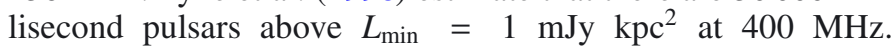
Desvignes (2009) finds 46000 millisecond pulsars above $L_{\min }=$ $0.1 \mathrm{mJy} \mathrm{kpc}^{2}$ at $1400 \mathrm{MHz}$ using model C of Lorimer et al. (2006), which amounts to 23000 above $0.2 \mathrm{mJy} \mathrm{kpc}^{2}$ with our adopted luminosity function. We adopted a total number of 30000 millisecond pulsars above $L_{\min }=0.2 \mathrm{mJy} \mathrm{kpc}{ }^{2}$ at $1400 \mathrm{MHz}$, which is a happy medium between the above estimates, using a spectral slope of -1.8 to convert $L_{\min }$ to the right frequency.

The observed distribution of millisecond pulsar rotation periods $P$ is a power law of index +3 (Hessels et al. 2007). However, the paucity of fast-spinning pulsars is the result of selection effects (see Sect. 1 and Lorimer et al. 1996). Following Lorimer et al. (1996), we adopt a distribution of rotation periods that is uniform in $\log (P)$. The lower limit of $0.65 \mathrm{~ms}$ is the lowest theoretically possible one (Haensel et al. 1995), and the upper limit reflects an observed cutoff. Cordes \& Chernoff (1997) assume a period distribution in the form $P^{-1}$. For the rotation periods of ordinary pulsars, a log-normal distribution is generally assumed (Lyne et al. 1985; Lorimer et al. 2006).

The distribution of luminosities is also modeled by a power law, down to the lowest luminosity (Lorimer et al. 2007; Hessels et al. 2007; Bagchi \& Lorimer 2011): $\rho(L)=L^{-n}$ if $L>L_{\min }$, and 0 otherwise, with $n$ around unity. We adopted $n=1$. The luminosity distribution of ordinary pulsars has been modeled by one or two power laws (Lyne et al. 1998; Guseinov et al. 2003). Models where the luminosity distribution depends on the pulsar period and its derivative have also been considered, for both millisecond and ordinary pulsars (Story et al. 2007). The flux 
density was computed by multiplying the luminosity by the square of the distance to the synthetic pulsar.

Finally, we take possible pulse smearing by the interstellar medium into account, using the Galactic free electron model NE20014 ${ }^{4}$ of Cordes \& Lazio (2002) to estimate the dispersion measure $(D M)$ at the distance of the synthetic pulsars. If $D M>86.38 P$, where $P$ is the period in $\mathrm{ms}$ and $D M$ is in $\mathrm{cm}^{-3} \mathrm{pc}$, the sensitivity is reduced by half. If $D M>172.76 P$, the sensitivity is reduced by a factor 3 or more, and we consider that the pulsar is undetectable (see Fig. 3). On the other hand, we ignore the effects on the flux density of diffractive interstellar scintillation, which can make the measured flux density vary by a large factor, as shown for PSR B1937+21 in Fig. 1.

\subsection{Results of the simulations}

We did many simulations of the distribution and detectability of the pulsar population in the region of our blind survey, using the above model and parameters. Out of a population of 30000 synthetic pulsars in the Galaxy, only about 4600 shortperiod millisecond pulsars $(P \leq 3 \mathrm{~ms})$ fall in the region of the survey extended to $0^{\circ}<l<120^{\circ}$ and $|b|<4^{\circ}$ for comparison with Fig. 4. There are about 5700 long-period millisecond pulsars $(P>3 \mathrm{~ms})$ in that region, which thus contains a bit more than a third of the total population of synthetic pulsars. We show the result of one simulation in Fig. 5. The distribution of the short-period pulsars is shown in the top panel, together with the histogram of flux densities above $0.1 \mathrm{mJy}$. The smooth curve is the mean histogram for a thousand simulations. The pulsars are concentrated in the region nearest to the Galactic center.

However, when considering the effect of high $D M$, that is, eliminating all the pulsars above the top diagonal line of Fig. 3, the overall population of detectable pulsars in the region of the survey is reduced by a factor 6 . This is shown in the bottom panel of Fig. 5, where the region near the Galactic center is no longer heavily populated, and the region $40^{\circ}<l<80^{\circ}$ becomes the most populated. This depopulation of the detectable pulsars is not so critical if one only considers the pulsars with a high flux density, say above $1 \mathrm{mJy}$. This population is reduced from 17 to 9, that is, by about half, because the depopulation affects mainly pulsars with a high $D M$, which are necessarily more distant, thus fainter and more difficult to detect.

The number of detectable pulsars for a given sensitivity cannot be read directly from the histogram of the lower panel of Fig. 5, because the actual sensitivity will depend on the $D M$ at the distance of the pulsar. Taking the example of our project, we find that 4.2 pulsars in the extended region of our survey are detectable for the nominal sensitivity of $1.7 \mathrm{mJy}$ of our last setup (taking the effect of large DM into account), and 7.7 pulsars are detectable for a sensitivity of $0.9 \mathrm{mJy}$ (which is achieved with four integrations of $160 \mathrm{~s}$ and reduction in "cluster mode"). This means that, if we want to double our chances of detecting a pulsar, we have to increase our sensitivity twofold. This can be achieved by a larger bandwidth, but, if keeping the equipment unchanged, it would require increasing the telescope time fourfold, which is not an economical strategy. It is more efficient to extend the region of the survey rather than increase the sensitivity (provided the added region is as rich in potential pulsars as the initial region). Only at much higher sensitivities does the number of synthetic pulsars go up significantly with increased sensitivity (see histograms of Fig. 5).

\footnotetext{
${ }^{4}$ http://rsd-www.nrl.navy.mil/7213/lazio/ne_model
}

Table 5. Probability of detecting short-period pulsars.

\begin{tabular}{lccc}
\hline \hline$N_{\mathrm{p}}$ & $P_{\mathrm{s}}(\%)$ & $P_{1.7}(\%)$ & $P_{0.9}(\%)$ \\
\hline 0 & 0.763 & 0.213 & 0.697 \\
1 & 0.151 & 0.223 & 0.174 \\
2 & 0.055 & 0.184 & 0.073 \\
3 & 0.020 & 0.145 & 0.035 \\
4 & 0.006 & 0.099 & 0.014 \\
5 & 0.003 & 0.059 & 0.004 \\
6 & 0.0006 & 0.038 & 0.0014 \\
7 & 0.0002 & 0.020 & 0.0006 \\
\hline
\end{tabular}

Now, to estimate whether a synthetic pulsar is detected in any one of our pointings, we model the beam pattern of the radiotelescope by a $\sin c^{2}$ function in both coordinates (modeling by a Gaussian is only acceptable within the half-power beamwidth), and compute the sensitivity correction at that pointing as $\Delta s=$ $\operatorname{sinc}^{2}(\mathrm{~d} \alpha) \operatorname{sinc}^{2}(\mathrm{~d} \delta)$, where $\mathrm{d} \alpha$ and $\mathrm{d} \delta$ are the angular distances of the synthetic pulsar to the center of the beam in right ascension and declination, respectively. The half-power beamwidth is $3.6^{\prime} \times 22^{\prime}$ at $1400 \mathrm{MHz}$ and zero declination, and there is an additional correction in declination above $22.7^{\circ}$. The beamwidth is about a factor 1.32 larger in declination at our highest declination. We also consider the loss of gain due to variable aperture efficiency and atmospheric absorption, using the equation given by Fouqué et al. (1990, their Eq. (1)).

The results of 200000 simulations are given in Table 5, where $N_{\mathrm{p}}$ is the number of detected synthetic pulsars, $P_{\mathrm{s}}$ the probability of detecting $N_{\mathrm{p}}$ pulsars in our survey of 5487 pointings, $P_{1.7}$ the probability of detecting $N_{\mathrm{p}}$ pulsars if we had completed the whole survey with a nominal sensitivity of $1.7 \mathrm{mJy}$ from beginning to end, and $P_{0.9}$ the probability of detecting $N_{\mathrm{p}}$ pulsars if we had surveyed the more densely populated region $|b|<2^{\circ}$ and $45.0^{\circ}<l<68.0^{\circ}$ (3761 pointings) with a higher sensitivity of $0.9 \mathrm{mJy}$ (by doing four 160-s integrations at each pointing and reducing the data in "cluster mode").

In summary, we had one chance in four of detecting one or more short-period pulsars in our incomplete survey. Were we to redo the complete survey with the sensitivity reached in the last setup, we would have a $79 \%$ chance of success, but even so the harvest would be far from spectacular. And this ignores the effect of interstellar scintillation, which reduces our chances of success by an unknown amount. While the third option of surveying a smaller region with a higher sensitivity may a priori seem attractive, it has a low return and is in practice unfeasible at a transit instrument like the Nançay radiotelescope, because it would monopolize the right-ascension slot $19 \mathrm{~h}-20 \mathrm{~h}$ for about two years.

As mentioned in Sect. 5.1, we miss nearby high-latitude millisecond pulsars when limiting the blind survey to low latitudes. If we set no limit in $b$ (there is of course no way of limiting the survey in linear height above the Galactic plane), we find that there are about 6510 (rather than 4600) short-period millisecond pulsars in the region of our survey, 1980 (rather than 730) detectable ones, and 29 (rather than 9) above 1 mJy. However, we would have to scan about half the sky visible at Nançay to complete the survey, so this is another unrealistic option.

Comparing our simulations with the population of known millisecond pulsars, we find 14 known field millisecond pulsars with a known flux density at $1400 \mathrm{MHz}$ in the region of our blind survey, and the faintest one has a flux density of $0.05 \mathrm{mJy}$. Down to that value, there are about 33 synthetic millisecond pulsars, so that about half the total population 
Table 6. List of observed faint polarized point-like radiosources.

\begin{tabular}{|c|c|c|c|c|c|c|c|c|c|}
\hline Name & $\begin{array}{c}\text { S1400 } \\
\text { mJy }\end{array}$ & $\%$ pol & $\begin{array}{c}\text { Date } \\
\text { yyyymmdd }\end{array}$ & $N$ & Name & $\begin{array}{c}\text { S1400 } \\
\text { mJy }\end{array}$ & $\%$ pol & $\begin{array}{c}\text { Date } \\
\text { yyyymmdd }\end{array}$ & $N$ \\
\hline NVSS J002145+744454 & 9.7 & 26.0 & 20080905 & 3 & NVSS J061712+260152 & 7.5 & 27.5 & 20081103 & 4 \\
\hline NVSS J003706+515737 & 7.3 & 40.7 & 20080905 & 3 & NVSS J070822-151518 & 10.0 & 26.1 & 20081204 & 3 \\
\hline NVSS J004419+514533 & 2.9 & 84.5 & 20080905 & 3 & NVSS J071545+051635 & 8.5 & 24.9 & 20081012 & 4 \\
\hline NVSS J012635+704215 & 4.4 & 76.8 & 20080905 & 4 & NVSS J071933-365933 & 7.6 & 26.4 & 20081106 & 3 \\
\hline NVSS J012705+703605 & 3.5 & 75.1 & 20080909 & 3 & NVSS J072438-271618 & 5.0 & 43.0 & 20081106 & 4 \\
\hline NVSS J012818+704655 & 5.3 & 64.0 & 20080909 & 3 & NVSS J072501+004534 & 7.5 & 41.6 & 20081204 & 4 \\
\hline NVSS J012932+701545 & 4.2 & 50.5 & 20080905 & 4 & NVSS J073949+004434 & 7.1 & 28.6 & 20081106 & 4 \\
\hline NVSS J013201+704729 & 3.3 & 113.3 & 20080909 & 4 & & & & 20081204 & 4 \\
\hline NVSS J030809+512443 & 7.4 & 32.7 & 20081003 & 3 & NVSS J082008-363214 & 6.3 & 34.3 & 20080703 & 3 \\
\hline NVSS J034707+402127 & 9.4 & 28.2 & 20081003 & 4 & NVSS J082838-380231 & 3.4 & 68.2 & 20080803 & 4 \\
\hline NVSS J035429+643653 & 9.5 & 22.5 & 20081001 & 3 & NVSS J083035-312745 & 6.5 & 32.8 & 20080703 & 3 \\
\hline NVSS J042204+363943 & 9.3 & 30.6 & 20081001 & 3 & NVSS J090355-384439 & 3.0 & 67.3 & 20080703 & 4 \\
\hline NVSS J043739+301858 & 5.9 & 66.3 & 20081001 & 4 & NVSS J155955-382320 & 6.0 & 35.3 & 20080911 & 3 \\
\hline NVSS J050224+250015 & 5.1 & 43.3 & 20081001 & 4 & NVSS J160116-381526 & 5.4 & 68.1 & 20080805 & 3 \\
\hline NVSS J052352+164533 & 5.2 & 38.7 & 20081210 & 3 & NVSS J162707-364109 & 3.9 & 55.1 & 20080805 & 3 \\
\hline NVSS J052906+215305 & 6.7 & 59.7 & 20081212 & 3 & NVSS J162943-340416 & 8.4 & 27.3 & 20080911 & 3 \\
\hline NVSS J053220+163421 & 8.8 & 22.8 & 20081103 & 3 & NVSS J163550-375558 & 4.7 & 54.9 & 20080706 & 3 \\
\hline NVSS J053959+19 & 5.4 & 37.6 & 20081113 & 3 & NVSS J164751-341010 & 4.2 & 48.3 & 20080805 & 4 \\
\hline NVSS J05 & 2.5 & 112.4 & 20081011 & 3 & NVSS J165146-353221 & 5.1 & 39.8 & 20080706 & 4 \\
\hline $134+302901$ & 9.5 & 22.4 & 20081210 & 4 & NVSS J165753-305936 & 5.1 & 52.2 & 20080911 & 4 \\
\hline $31+500635$ & 4.1 & 49.5 & 20081212 & 4 & NVSS J194518+101520 & 9.9 & 20.2 & 20080809 & 3 \\
\hline NVSS J054456+491607 & 5.0 & 47.2 & 20081103 & 4 & NVSS J195016+404408 & 4.9 & 44.9 & 20080807 & 3 \\
\hline NVSS J054532+215524 & 8.7 & 24.7 & 20081011 & 3 & NVSS J200102+420928 & 4.6 & 100.7 & 20080807 & 3 \\
\hline NVSS J054650+495147 & 6.3 & 44.3 & 20081113 & 4 & NVSS J200109+112314 & 8.4 & 26.8 & 20080809 & 3 \\
\hline NVSS J054747+392734 & 9.3 & 23.9 & 20081210 & 4 & NVSS J202139+233729 & 4.1 & 54.1 & 20080809 & 4 \\
\hline NVSS J055120+032729 & 8.2 & 26.3 & 20081212 & 4 & NVSS J203453+214620 & 4.6 & 88.7 & 20080807 & 4 \\
\hline NVSS J055600+185916 & 9.1 & 27.5 & 20081103 & 4 & NVSS J204040+304747 & 3.5 & 62.9 & 20080711 & 3 \\
\hline NVSS J060348+215030 & 3.6 & 57.2 & 20081011 & 4 & NVSS J210904+382547 & 7.8 & 26.0 & 20080711 & 3 \\
\hline NVSS J060348+343522 & 10.0 & 22.0 & 20081113 & 4 & NVSS J211354+611530 & 3.7 & 61.4 & 20080711 & 4 \\
\hline NVSS J060411+311018 & 9.7 & 21.8 & 20081212 & 4 & NVSS J215324+395318 & 8.6 & 24.0 & 20080711 & 4 \\
\hline NVSS J060820+171726 & 8.0 & 26.0 & 20081210 & 4 & NVSS J223844+451415 & 10.0 & 20.4 & 20080708 & 3 \\
\hline NVSS J061011+150106 & 7.4 & 28.8 & 20081011 & 4 & NVSS J230058+713519 & 9.8 & 26.4 & 20080708 & 3 \\
\hline NVSS J061040+332755 & 8.6 & 24.1 & 20081113 & 4 & NVSS J234546+544241 & 8.2 & 24.9 & 20080708 & 4 \\
\hline
\end{tabular}

predicted by our simulations in that region have already been detected.

Incidentally, the sensitivity of our blind survey is such that, among these 14 known millisecond pulsars, only PSR B1937+21 and PSR J2007+2722 (which has a period of $24 \mathrm{~ms}$ ) can be detected with our setup.

To put the results of our blind survey in perspective, we mention the results of another blind survey for millisecond pulsars conducted at Nançay at about the time that we started our own survey. A survey of 45000 pointings in the region $180^{\circ} \geq l \geq$ $-15^{\circ}$ and $|b| \leq 3^{\circ}$ was conducted in 1997-98 (Foster et al. 1997), using a different backend and method of analysis. The frequency, bandpass, integration time, and nominal sensitivity of the survey were $1360 \mathrm{MHz}, 144 \mathrm{MHz}, 120 \mathrm{~s}$, and $0.6 \mathrm{mJy}$, respectively, and the sampling resolution was $1.5 \mathrm{MHz}$ in frequency and $60 \mu \mathrm{s}$ in time. It produced only two new pulsars, both with periods longer than $100 \mathrm{~ms}$ (Ray et al. 1999). The reanalysis of $15 \%$ of the stored data with a different method produced 341 candidate millisecond pulsars, and observing 200 of them again did not reveal any new pulsar (Desvignes 2009). With our simulations, we find that there are 30 synthetic millisecond pulsars in the region of the survey above the flux limit, but only 14 of them are detectable with our processing method in the best-case scenario (i.e. if the telescope points exactly at the pulsar in each case). This confirms what was probably widely believed, that it is notoriously difficult to detect millisecond pulsars. As one referee of our telescope time requests stated, it is a high-risk, high-reward project.

\section{Conclusions}

The present project was started with no assumptions on the total population of millisecond pulsars (except that it must be large) and with the specific aim of detecting a population of fastspinning (sub)millisecond pulsars. The results of the small surveys targeted at unidentified radiosources suggest that, despite PSR B1937+21, bright fast-spinning pulsars are rare. The null result of the blind survey agrees with our present understanding of the field population of millisecond pulsars, the order of magnitude of the total population, its absence of strong clustering, and the distribution of periods. Our conclusion is that the present models of the Galactic population of millisecond pulsars are not unduly pessimistic and that the population of fast-spinning millisecond pulsars cannot be unexpectedly large.

This project was also fruitful in a broader context: over 24 students worked as interns on the project over the years, and most of them subsequently made use of the acquired skills in their career.

Finally, we hope that future prospective searches for millisecond pulsars will successfully make use of our experience and of the numbers given here.

Acknowledgements. We first wish to acknowledge the help of numerous students who contributed to this project, mainly on its technical aspects, over the years: Vincent Galeote (1993), Jérôme Moueza (1994-95), Jean-Christophe Noël (1994-95), Yves Rutschlé (1995), Eric Soudanas (1995), David Descazaux (1995), Yannick Magnaud (1995), Olivier Danet (1996-97), Bénédicte Rougeaux (1995-99), Gérald Giraud (1996), Paul Thierry (1998), Sebastian Karl (1999), Lionel Aubépart (2000), Benoit de Vilèlle (2001), 
Mael Guennou (2002), Miguel Charcos (2002), Sébastien Rosete (2003), Franck-Sylvain Petit (2004), Julien Badroudine (2005), Fabien Bricout (2005), Angel Boukhari (2005), Alexandre Durand (2006), Amin Shahsavar (2006), and Guillaume Carrié (2008). This project was supported until 1998 by Centre National d'Etudes Spatiales. We thank the staff of the Nançay radiotelescope for assistance in setting up and testing our dedicated backends, and Ismaël Cognard for help with interfacing our backend with the telescope and for letting us use the cluster of PCs for data analysis. We are grateful to Jean-Michel Martin for giving us some director's time on the radiotelescope for examining the feasability of our search for millisecond pulsars in globular clusters, to Pierre Colom for information on the geometry of the telescope beam, to the anonymous referee for useful comments that helped improve the paper, and to the language editor for improving the style of the paper.

\section{References}

Aulbert, C. 2007, MPE Report 291, 216

Bagchi, M., \& Lorimer, D. R. 2011, AIP Conf. Proc., 1357, 173 [arXiv: 1012.4705]

Bailyn, C. D. 1995, ARA\&A, 33, 133

Cordes, J. M., \& Chernoff, D. F. 1997, ApJ 482, 971

Cordes, J. M., \& Lazio, T. J. W. 2002 [arXiv: astro-ph/0207156]

Crawford, F., Kaspi, V., \& Bell, J. 2000, Pulsar Astronomy, ASP Conf. Ser., 202, 31

D'Amico, N. 2000, Pulsar Astronomy, ASP Conf. Ser., 202, 27

De Breuck, C., van Breugel, W., Rottgering, H. J. A., \& Miley, G. 2000, A\&AS, 143,303

Desvignes, G. 2009, Thèse de doctorat de l'Université d'Orléans

Edwards, R. T., van Straten, W., \& Bailes, M. 2001, ApJ, 560, 365

Faucher-Giguère, C.-A., \& Kaspi, V. M. 2006, ApJ, 643, 332

Faucher-Giguère, C.-A., \& Loeb, A. 2010, JCAP, 1, 5

Foster, R. S., Ray, P. S., Cadwell, B. J., et al. 1997, BAAS, 29, 1392

Fouqué, P., Durand, N., Bottinelli, L., Gouguenheim, L., \& Paturel, G. 1990, A\&AS, 86, 473

Freire, P. C. C., Hessels, J. W. T., Nice, D. J., \& Ransom, S. M. 2005, ApJ, 621, 959

Guinot, B., \& Arias, E. F. 2005, Metrologia, 42, S20

Guseinov, O. H., Yazgan, E., Tagieva, S. O., \& Özkan, S. 2003, RMxAA, 39, 267
Haensel, P., Salgado, M., \& Bonazzola, S. 1995, A\&A, 296, 745

Han, J. L., Manchester, R. N., Lyne, A. G., \& Qiao, G. J. 2004, IAU Symp., 218 , 135

Hessels, J. W. T., Ransom, S. M., Stairs, I. H., Kaspi, V. M., \& Freire, P. C. C. 2007, ApJ, 670, 363

Hessels, J. W. T., Ransom, S. M., Kaspi, V. M., et al. 2008, AIPC, 983, 613

Hut, P., McMillan, S., Goodman, J., et al. 1992, PASP, 104, 981

Janssen, G. H., Stappers, B. W., Bassa, C. G., et al. 2010, A\&A, 514, A74

Lattimer, J. M., \& Prakash, M. 2007, Phys. Rep., 442, 109

Lorimer, D. R. 2008, Liv. Rev. Rel., 11, 8

Lorimer, D. R., Nicastro, L., Lyne, A. G., et al. 1995, ApJ, 439, 933

Lorimer, D. R., Lyne, A. G., Bailes, M., et al. 1996, MNRAS, 283, 1383

Lorimer, D. R., Faulkner, A. J., Lyne, A. G., et al. 2006, MNRAS, 372, 777

Lorimer, D. R., McLaughlin, M. A., Champion, D. J., \& Stairs, I. H. 2007, MNRAS, 379, 282

Lyne, A. G., Manchester, R. N., \& Taylor, J. H. 1985, MNRAS, 213, 613

Lyne, A. G., Brinklow, A., Middlewitch, J., et al. 1987, Nature, 328, 399

Lyne, A. G., Manchester, R. N., Lorimer, D. R., et al. 1998, MNRAS, 295, 743

Manchester, R. N. 2011, [arXiv: 1101.5202]

Manchester, R. N., Hobbs, G. B., Teoh, A., \& Hobbs, M. 2005, AJ, 129, 1993

Navarro, J., De Bruyn, A. G., Frail, D. A., Kulkarni, S. R., \& Lyne, A. G. 1995, ApJ, 455, L55

Petit, G., \& Tavella, P. 1996, A\&A, 308, 290

Possenti, A., D’Amico, N., Manchester, R. N., \& Camilo, F. 2003, ApJ, 599, 475

Ray, P. S., Cadwell, B. J., Lazio, T. J. W., et al. 1999, BAAS, 31, 903

Ransom, S. M., Hessels, J. W. T., Stairs, I. H., et al. 2005, Science, 307, 892

Rougeaux, B. 1999, Thèse de doctorat de l'Université Paul Sabatier

Rougeaux, B., Petit, G., Fayard, T., \& Davoust E. 2000, Exp. Astron., 10, 473

Shi, H., Liang, H., Han, J. L., \& Hunstead, R. W. 2010, MNRAS, 409, 821

Stappers, B. W., Bailes, M., Lyne, A. G., et al. 1996, ApJ, 465, L119

Story, S., Gonthier, P. L., \& Harding, A. K. 2007, ApJ, 671, 713

Taylor, J. H. 1991, Proc. IEEE, 79, 1054

van Haasteren, R., Levin, Y., McDonald, P., \& Lu, T. 2009, MNRAS, 395, 1005

Verbiest, J. P. W., Bailes, M., Coles, W. A., et al. 2009, MNRAS, 400, 951

Yan, W. M., Manchester, R. N., van Straten, W., et al. 2011, MNRAS, 414, 2087

Yusifov, I. 2004, in The Magnetized Interstellar Medium, ed. B. Uyaniker et al. (Katlenburg-Lindau: Copernicus GmbH), 165

Yusifov, I., \& Küçük, I. 2004, A\&A, 422, 545 